\title{
Tissue microarray: an emerging potential research tool
}

\begin{abstract}
Tissue microarray (TMA) is an amazing reward to the researchers and proved to be a boon to the field of Research and Pathology. Through this technology, thousands of samples can be analysed simultaneously thus saving time and manpower. It also opens windows to other techniques like immunohistochemistry, in situ hybrid dization, DNA, RNA and protein expression, cell assays etc. TMA has got several benefits against meager disadvantages. It is a versatile technique with definitive clinical and pathological applications.
\end{abstract}

Volume 2 Issue $3-2017$

\author{
Megha Jain,' Anish Gupta,' Samar Khan² \\ 'Department of Oral Pathology and Microbiology, Peoples \\ Dental Academy, India \\ ${ }^{2}$ Department of Oral and Maxillofacial Pathology, Jazan \\ University, Saudi Arabia
}

Correspondence: Megha Jain, Department of Oral Pathology and Microbiology, Peoples Dental Academy, Bhopal-462037,

Madhya Pradesh, India, Email megha.vipin 12@gmail.com

Received: June 22, 2017 | Published: July 19, 2017

\section{Introduction}

Tissue microarray [TMA] is a latest novelty in the area of pathologies and biomedical researches. The method was designed as a high-throughput molecular device that permits analysis of expression of several biomarkers simultaneously on thousands of tissue samples. ${ }^{1,2}$ Rationalisation of biomarkers using the conventional histopathological procedure is time consuming, laborious and pricey especially, when several markers are to be tested on various specimens. So, TMA is expected to surmount these substantial problems. ${ }^{3}$ Tissue microarrays are produced by harvesting cylindrical tissue cores from different paraffin donor blocks and re-embedding them in an array on a single recipient paraffin block such that multiple specimens can be analyzed at the same time thus, conserving resources by analysis of small-core biopsies of blocks rather than complete sections. ${ }^{1,4}$

\section{Types of tissue microarray}

\section{Based upon their material of origin}

I. Tissue microarrays -if they are constructed from paraffin embedded material. This technique offers fine morphological details but can compromise antigenicity. ${ }^{5}$

II. Resin Tissue microarrays- If the tissues are arrayed into a resin recipient block like glycol methacrylate resin. Resin tissue micro arrays provides enhanced tissue morphology along with good antigenicity and thin section preparation. It could be used as an better substitute for frozen and formaline fixed and paraffin embedded (FFPE) TMAs. ${ }^{5,6}$

III. Cryoaarays- if they are constructed from frozen tissue. This newer technique was developed to overcome difficulty associated with antigenic alteration and mRNA degradation in case of FFPE tissues. Frozen tissues embedded in OCT compounds proved to give enhanced results with DNA, RNA and protein analyses.

IV. TMAs have also been constructed from paraffin embedded cell lines or cell blocks.

Ferrer $\mathrm{B}$ et al. ${ }^{8}$ proposed and validated that paraffin-embedded cell line microarrays (PECLIMA) allow concurrent expression of several antigens in multiple cell lines under diverse experimental setting. This method is compatible with immunocytochemistry and in situ hybridization technique. Advantage associated being low cost and long term storage. ${ }^{8}$ Cell Block microarray technique proved to be very simple and cost-effective and its construction doesn't necessitate any complex tools. ${ }^{9}$

\section{Based upon their applications $\mathbf{s}^{5,10}$}

I. Predictive TMAs: purpose is to authenticate biomarkers that envisage treatment response.

II. Control tissue TMAs: purpose is to set protocols for researches and experimental analysis.

III. TMAs for validation of markers.

IV. Prognostic TMAs: used to check association between staining results and clinical endpoints.

V. Progression TMAs: purpose is to keep check on tumor development and progression. Thus, can reduce cancer incidence and mortality For Eg: Progression TMAs for colon will be normal colon, Dysplastic (low and high grade) adenomas and adenocarcinomas. ${ }^{11}$

\section{Applications of tissue microarray 1,2,4,10,12-17}

I. TMA technology allows parallel conventional and molecular profiling thereby enabling morphology, DNA, RNA and protein targets to be analysed on multiple tissue samples.

II. TMA can be utilized for carrying out large-scale analyses using immunohistochemistry (IHC) and insitu hybridization (ISH)

III. TMAs significantly aids in rendition of molecular alteration into clinical upshot.

IV. Helps in identification of new diagnostic and prognostic disease markers especially cancer researches.

V. TMAs can be used to associate molecular alterations with the specific stage of tumor progression.

VI. TMAs assist in testing and standardization of potential therapeutic targets. 
VII. TMAs have also been utilized in Xenograft tumor assays and Tissue immunoblotting.

VIII. Facilitates retrospective and prospective human and animal tissue researches and cell line cytospin cell block studies.

IX. TMAs prove to facilitate IHC staining procedure and interpretation of external and internal quality control. TMAs surmount intralaboratory and interlaboratory variation owing to differences in antigen retrieval, staining protocols, antibodies used and in interpretation of results.

X. Originally TMA technology was used in cancer researches but, presently its scope is expanding and reported in non-neoplastic pathologies such as neurodegenerative diseases, dermatological, cardiac and placental diseases are also beginning to emerge.

\section{Tissue microarray: Benefits and pitfalls}

Advantages of TMAs of course outnumber its limitations ${ }^{7,16-18}$

I. TMAs are time efficient compared to traditional practice.

II. Analyses of genomic and biomarker expression on up to thousand of samples simultaneously.

III. Conservation of tissue resources and experimental reagents.

IV. Quantitative amplification of the sample tissues which are normally sparsely available in the pathology labs.

V. TMA has been applied to tumor researches such as glioma, breast tumor, lung cancer, oral cancer etc.

VI. Experimental uniformity i.e each tissue sample is treated in an identical manner.

VII. Frozen tissue microarrays have significant advantages over paraffin embedded tissue related to antigenic alteration and degradation.

VIII. TMAs format provides opportunities for digital imaging acquisition, image processing and database integration.

\section{The reflex criticisms of TMA technique are:}

I. Small tissue cores harvested may not be representative of the entire tumor mass, chiefly heterogenous cancer or in case of antigens with heterogeneous staining patterns. ${ }^{1,4}$ A tumor tissue may consist of diverse histological areas within like areas of apoptosis, necrosis or increased proliferation etc and therefore it is not possible to sample all areas in single tissue core. ${ }^{19}$ However this problem can be stamp out by using larger punch needles.

II. TMA equipments are quite expensive which further limits its utilization as a routine practice in many countries. ${ }^{20}$

III. Tissue may be lost during processing which is attributed to small size of tissue cores. ${ }^{5}$

IV. Thorough knowledge and practice required to analyse complex datasets of TMAs. ${ }^{5}$

V. Construction of tissue arrays mandate technical skills and expertise. ${ }^{12}$

However, alternatives are continuously evolving to circumvent these problems associated with TMAs.

\section{Conclusion}

Tissue microarrays proved to be multifaceted and efficient technology in the field of Research and Pathology. TMA system hastens transition of crucial research findings into clinical applications. Massive data obtained through the TMAs will definitely give potential scope to researchers and scholars to further carry out studies on large platforms.

\section{Acknowledgements}

None.

\section{Conflict of interest}

The author declares no conflict of interest.

\section{References}

1. Jawhar NMT. Tissue Microarray: A rapidly evolving diagnostic and research tool. Ann Saudi Med. 2009;29(2):123-127.

2. Giltnane JM, Rimm DL. Technology insight: Identification of biomarkers with tissue microarray technology. Nat Clin Pract Oncol. 2004;1(2):104-111.

3. Shergill IS, Shergill NK, Arya M, et al. Tissue microarrays: a current medical research tool. Curr Med Res Opin. 2004;20(5):707-712.

4. Rimm DL, Camp RL, Charette LA, et al. Tissue microarray: a new technology for amplification of tissue resources. Cancer J. 2001;7(1):24-31.

5. Parsons M, Grabsch H. How to make tissue microarrays. Diagnostic Histopathology. 2009;15(3):142-150.

6. Howat WJ, Warford A, Mitchell JN, et al. Resin tissue microarrays: a universal format for immunohistochemistry. J Histochem Cytochem. 2005;53(10):1189-1197.

7. Fejzo MS, Slamon DJ. Frozen tumor tissue microarray technology for analysis of tumor RNA, DNA, and Proteins. Am J Pathol. 2001;159(5):1645-1650

8. Ferrer B, Bermudo R, Thomson T, et al. Paraffin-embedded cell line microarray (PECLIMA): development and validation of a highthroughput method for antigen profiling of cell lines. Pathobiology. 2005;72(5):225-232.

9. Wen $\mathrm{CH}, \mathrm{Su}$ YC, Wang SL, et al. Application of the microarray technique to cell blocks. Acta Cytol. 2007;51:42-46.

10. Kallioniemi OP, Wagner U, Kononen J, et al. Tissue microarray technology for high-throughput molecular profiling of cancer. Hum Mol Genet. 2001;10(7):657-662.

11. Chen WC, Lin MS, Zhang BF, et al. Survey of molecular profiling during human colon cancer development and progression by immunohistochemical staining on tissue microarray. World J Gastroenterol. 2007;13(5):699-708.

12. Kumar B, De Silva M, Venter DJ, et al. Tissue microarrays: a practical guide. Pathology. 2004;36(4):295-300.

13. Al Kuraya K, Simon R, Sauter G. Tissue microarrays for high-throughput molecular pathology. Ann Saudi Med. 2004;24(3):169-174.

14. Wang H, Zhang W, Fuller GN. Tissue microarrays: applications in neuropathology research, diagnosis, and education. Brain Pathol. 2002;12(1):95-107.

15. Skacel M, Skilton B, Pettay JD, et al. Tissue microarrays: a powerful tool for high-throughput analysis of clinical specimens: a review of the method with validation data. Appl Immunohistochem Mol Morphol. 2002;10(1):1-6. 
16. Avninder S, Ylaya K, Hewitt SM. Tissue microarray: A simple technology that has revolutionized research in pathology. J Postgrad Med. 2008;54:158-162.

17. Watanabe A, Cornelison R, Hostetter G. Tissue microarrays: applications in genomic research. Expert Rev Mol Diagn. 2005;5(2):171-181.

18. Zhang X, Su D, Cheng Q. Advantages and applications of tissue microarray technology on cancer research. Chinese Journal of Cancer Research. 2003;15(1):74-78.
19. Packeisen J, Korsching E, Herbst H, et al. Demystified... Tissue microarray technology. J Clin Pathol Mol Pathol. 2003;56:198-204.

20. Singh A, Sau AK. Tissue Microarray: A powerful and rapidly evolving tool for high throughput analysis of clinical specimens. IJCRI. 2010;1(1):1-6. 